\title{
Clinical Pharmacology and Vascular Risk
}

\author{
G. Silvestrelli*,1, F. Corea ${ }^{2}$, S. Micheli ${ }^{3}$ and A. Lanari*,1 \\ ${ }^{1}$ Stroke Unit, Section of Neurology, C. Poma Hospital, Mantova, Italy \\ ${ }^{2}$ UO Gravi Cerebrolesioni, Odpedale San Giovanni Battista, Foligno, Italy \\ ${ }^{3}$ Stroke Unit, Division of Cardiovascular Medicine, University of Perugia, Perugia, Italy
}

\begin{abstract}
Pharmacological treatment and several drugs of abuse have been associated with ischemic heart disease (IHD) and cerebrovascular diseases (CVD). However, there is a paucity of data on the independent risk of vascular disease (VD) associated with pharmacological treatment and no controlled trials demonstrating a reduction in risk with abstinence.

Information about IHD and CVD-related drug abuse is mainly limited to epidemiological studies focused on urban populations. The potential link between some pharmacological treatments (estrogen, some oncologic drugs and some atypical antipsychotics) and cerebrovascular adverse events was analyzed, but disagreement about an association persists. Drugs of abuse, including cocaine, amphetamines and heroin, have been associated with an increased vascular risk.

These drugs can cause abrupt changes in blood pressure, vasculitic-type changes, lead to embolization caused by infective endocarditis, and hemostatic and hematologic abnormalities that can result in increased blood viscosity and platelet aggregation. Long-term treatment strategies based on medication, psychological support, and outreach programs play an important role in treatment of drug dependency.

In these last years public interest in risk factors for VD has been constantly increasing and the successful identification and management of pharmacological treatment and drug abuse can be challenging. One of the major public health issues for the future will be to focus more on new vascular risk factor recognition and management.

The objective of this chapter is to review the relevance of IHD and CVD associated with various pharmacological treatments and drug abuse with focusing on ischemic disease.

This chapter reports the clinical evidence of this association and analyzes the experimental role of new drugs as a growing risk factor of VD with the hypothetical new association. In conclusion, in this chapter great attention is paid to evaluating the scientific and real evidence of cerebrovascular effect and drug use and abuse so as to identify a new groups of "modifiable" risk factors.
\end{abstract}

Keywords: Risk factor, pharmacological treatment, drug abuse, stroke.

\section{INTRODUCTION}

VD is the major cause of death and disability worldwide. Strategies to reduce the incidence of VD include prevention of first-ever and recurrent VD. One of the main strategies of stroke and IDH prevention is the "high risk" approach which aims to reduce VD by identifying individuals at high vascular risk and lowering their risk by means of optimal medical therapies. VD prevention is a crucial issue and consists of an optimal management of vascular risk factors.

Most patients with recurrent VD had multiple risk factors identified at the time of their first stroke or/and IDH, but most of these risk factors were either undertreated or untreated. A smaller number of patients had newly identified vascular risk factors at admission. Epidemiological studies have advanced our understanding of new vascular risk factors and clinical trials have demonstrated effective interventions to decrease VD risk by modifying vascular risk factors.

*Address correspondence to these authors at the Stroke Unit, Section of Neurology, Carlo Poma Hospital, Lago Paiolo n. 10, 46100 Mantova, Italy; Tel: +39 0376-201686; Fax: +39 0376-201969;

E-mails: silvestrelli@hotmail.it, alessialanari@hotmail.com
The categories are expanding and include those related to infection, inflammation, sleep disorders, haemostasis, nutrition, endocrine, one's individual genotype, drugs of abuse and specific pharmacological treatment; these are nontraditional risk factors for VD in rapid expansion. Many of the promising factors lack large randomized prospective population studies confirming direct cause and effect.

The complexity of the pathophysiology of this disease remains a challenge.

\section{EXPERIMENTAL AND CLINICAL EVIDENCE OF PHARMACOLOGICAL VASCULAR RISK}

\section{Pathophysiology}

The aim of pathophysiology in VD is on the blood and/or endothelium disease. Vascular dysfunction underlies the pathophysiology of a wide range of diseases, including atherosclerosis, mechanical stress, smoke exposure, hypercholesterolemia, hyperhomocysteinemia and chronic infection (direct or indirect), diabetes and arthritis. The endothelium plays an important role in cardio and cerebrovascular homeostasis, either through the vascular tonus and its regulation, or through coagulation and the inflammatory response 
[1]. Endothelial dysfunction is implicated in the genesis of atherosclerosis and other chronic disorders, such as diabetes mellitus and hypertension. Abnormalities of the vascular endothelium contribute to all stages of atherosclerosis from lesion development to clinical IDH events. A variety of pharmacological and behavioural interventions have been shown to reverse endothelial dysfunction in patients with IDH. A large number of epidemiological studies suggest that dietary factors, including increased intake of flavonoidcontaining foods and beverages, reduce cardiovascular risk, and recent studies have shown that such beverages have favourable effects on endothelial function [2]. Angiogenic function is progressively impaired with increasing age and, therefore, has been linked to the increased risk of many of these diseases among older people.

Blood levels of inflammatory markers (e.g., C-reactive protein CPR, serum amyloid A, fibrinogen, plasma viscosity, erythrocyte sedimentation rate, leukocyte count and low serum albumin) have been associated with vascular risk factors and with prevalent and incident atherothrombotic cardiovascular disease (coronary heart disease, stroke and peripheral arterial disease). More recently, cytokines (e.g., interleukin6) and soluble adhesion molecules (e.g., intercellular adhesion molecule-1, vascular cell adhesion molecule-1) have been associated with both risk factors and disease [3]. Raised plasma fibrinogen levels are associated with an increased risk of vascular events. This may be mediated by adverse effects of fibrinogen on plasma viscosity, coagulation, platelet activity, inflammation and atherogenesis [4]. However, there is as yet no drug that specifically lowers plasma fibrinogen levels on a long-term basis. Some trials have demonstrated that lowering plasma fibrinogen levels will result in a decreased risk of vascular events [3]. However, such a trial may never happen unless a specific agent is discovered or designed. Several drugs that are used in VD prevention (e.g., lipid lowering agents and antihypertensives) may influence plasma fibrinogen levels. Whether such an additional effect accounts for variations in the benefit resulting from the use of different drugs within the same class remains to be established. The debate continues as to whether fibrinogen is just a marker of vascular risk or whether lowering its circulating levels will result in a significant decrease in clinically relevant endpoints. Whatever the case, the measurement of plasma fibrinogen levels is likely to provide a more comprehensive estimation of risk.

Unique drug targets have already been identified in some of these emerging vascular risk factors (Table $\mathbf{1}$ ).

Table 1. Clinical Pharmacological Vascular Risk Factors

\begin{tabular}{|c|c|}
\hline $\begin{array}{c}\text { Some Pharmacological } \\
\text { Treatment }\end{array}$ & Several Drugs of Abuse \\
\hline \hline sex hormone therapy & pharmacological drugs \\
\hline oral contraceptive use & cocaine, amphetamines and heroin \\
\hline antipsychotic drugs & \\
\hline thiazolidinediones & \\
\hline immunomodulatory drugs & \\
\hline antiepileptic drugs & \\
\hline COX-inhibitors & \\
\hline HMG-CoA reductase inhibitors & \\
\hline
\end{tabular}

\section{Sex hormone Therapy}

Menopause is a risk factor for VD because estrogen withdrawal has a detrimental effect on cardiovascular function and metabolism. Now there is evidence of an association between endothelial dysfunction and reduced endogenous production of estrogens after natural or surgical menopause or premature ovarian failure in women with or without coronary arterial disease [5]. The menopause compounds many traditional VD risk factors, including changes in body fat distribution from a gynoid to an android pattern, reduced glucose tolerance, abnormal plasma lipids, increased blood pressure, increased sympathetic tone, endothelial dysfunction and vascular inflammation.

The actions of endogenous estrogens on the cardiovascular system can be mediated directly on the vessels or indirectly through the modulation of cardiovascular risk factors, as well as on the lipid profile (reduction of total cholesterol and LDL, increase in HDL), as already described more than 20 years ago. More recently, studies have also demonstrated an antioxidant effect by estrogen, reducing LDL oxidation in vivo and in vitro [6]. The direct effects of estrogen on the vascular system which modulate_the vascular tonus comprise I) acute vasodilatation, increasing the synthesis and bioactivity of nitric oxide [7], II) long-term modulation of vascular tonus, regulating the production of prostaglandins and expression of eNOS and the endothelin gene [8], III) inhibition of endothelin-induced vasoconstriction [9], and IV) inhibition of sympathetic activity [8]. In addition to these actions on the vascular tonus, estrogen exerts an antiproliferative action on the vascular smooth layer [10]. It also appears to have a major role in vascular remodeling, inhibiting the proliferation of the inner layer after injury [11] and increasing the expression of contractile proteins in the myocardium [12]. Despite repeated evidence of estrogen-related neuroprotection, large population-based studies in postmenopausal women receiving estrogen replacement did not demonstrate the expected neuroprotection.

On the other hand, androgen and sex hormone-binding globulin levels have been associated with risk of cardiovascular disease in pre- and postmenopausal women [13]. An increase in circulating androgens appears to be associated with insulin resistance and a predictor of diabetes mellitus [14]. In previous studies a positive association, independent of obesity, was demonstrated between testosterone levels and hyperinsulinemia in postmenopausal women with no clinical evidence of VD [15]. In some tissues, such as the liver, estrogen can mediate both beneficial (expression of genes of apoproteins that improve the lipid profile) and adverse effects (increase in the expression of procoagulant factors and decrease of fibrinolytic factors) [4].

The pharmacological use of estrogen exerts influence on the circulating levels of markers of vascular tonus, and inflammation, as well as prothrombotic, and fibrinolytic markers, but the impact of these changes on the atherosclerotic disease is still uncertain. Clinical and animal studies suggest multiple cardiovascular effects of selective estrogen receptor modulators. For example, raloxifene lowers serum levels of cholesterol and homocysteine, attenuates oxidation of lowdensity lipoprotein, inhibits endothelial-leucocyte interaction, improves endothelial function and reduces vascular smooth muscle tone [13]. Available evidence suggests that 
raloxifene and tamoxifen are capable of acting directly on both endothelial cells and the underlying vascular smooth muscle cells and cause a multitude of favourable modifications of the vascular wall, which jointly contribute to improved local blood flow.

Observational studies and randomized clinical trials suggest that hormone replacement therapy (HRT) started soon after the menopause may confer cardiovascular benefit. In contrast to other synthetic progestogens used in continuous combined HRTs, the unique progestogen drospirenone has antialdosterone properties. Drospirenone can therefore counteract the water- and sodium-retaining effects of the estrogen component of HRT via the renin-angiotensin-aldosterone system, which may otherwise result in weight gain and raised blood pressure. As a continuous combined HRT with 17beta-estradiol, drospirenone has been shown to significantly reduce blood pressure in postmenopausal women with elevated blood pressure, but not in normotensive women. Therefore, in addition to relieving climacteric symptoms, drospirenone/17beta-estradiol may offer further benefits in postmenopausal women, such as improved CVD risk profile [16].

Estrogen deficiency causes progressive reduction in endothelial function. Despite the benefits of HRT evident in earlier epidemiological studies, recent randomized trials of HRT for the prevention of heart disease found no overall benefit. Instead, HRT users had higher incidences of stroke and heart attack. Although experimental data and results of observational studies suggest that HRT was associated with a reduction in the risk of heart disease, the results for stroke have been far less clear. Several recent randomized secondary prevention studies have found that HRT is not protective against the risk of coronary heart disease, stroke or progression of atherosclerosis. Moreover, an increased risk of stroke and first coronary event with hormone therapy has been found in low-risk healthy post menopausal women. In the light of these findings, although the absolute risk of vascular event associated with HRT is low, most organizations recommend caution in using HRT and finally in postmenopausal women, treatment of arterial hypertension and glucose intolerance should be priorities.

\section{Oral Contraceptive Use}

The most frequent major adverse effect of hormonal contraception is an increased risk of VD. The effect on the risk of venous thromboembolism, CVD and IHD differs and is strongly influenced by smoking and the presence of other cardiovascular risks factors, such as hypertension and diabetes mellitus. The incidence of each disease rises with age and there are differences in risk among hormonal contraceptive preparations.

Mortality rates for cardiovascular diseases are very low in women of reproductive age. IDH mortality rates rise from $<0.4$ per 100,000 woman-years at age 15-24 years to the range 2 to 7 per 100,000 woman-years at age 35-44 years. Stroke mortality rates similarly rise steeply with age and are between 3 and 5 times higher than those for IDH. Venous thromboembolism mortality rates rise less steeply with age and are approximately one-tenth the IDH mortality rates at age 35-44 years. The adverse effect of oral contraceptives (OC) on the risk of venous thromboembolism is the most important contributor to the total number of cardiovascular cases attributable to $\mathrm{OC}$ use. The increased risk of stroke and IDH dominate the patterns of mortality in OC users and smokers. The additional risks attributable to smoking are greater than the additional risks attributable to OC use. The risk attributable to OC use in women $<35$ years of age is small, even if they smoke, but there are substantially increased risks in older women who both smoke and use OC. The additional mortality attributable to $\mathrm{OC}$ use can be reduced by screening users, as this results in lower relative risks of ischemic stroke and IDH. Differences between OC types in the relative risk of venous thromboembolism contribute little to the total cardiovascular mortality associated with OC use, even though the total number of cardiovascular events is increased. A potential reduction in the risk of IDH with desogestrel and gestodene compared with levonorgestrel-containing OC would have little difference on overall mortality rates in women in their 20 s and 30 s, but may result in a net reduction in OC-attributable mortality in women aged 40-44 years who smoke. An overall quantification of the risks for different types of oral contraceptive users is necessary for an informed choice of contraceptive method, and any assessment of the balance of cardiovascular risks is complex. The model provides a tool to assess, at the level of the individual, the risks associated with use of different OC according to personal circumstances. It is important to consider the user's age and smoking status when determining OC attributable risks (17). Estrogen plus progestin was associated with doubling the risk of venous thrombosis. Estrogen plus progestin therapy increased the risks associated with age, overweight or obesity, and factor V Leiden (18).

Much of the perceived increased stroke risk associated with the use of OCs is based on early studies with high-dose preparations (ie, first-generation OCs containing $\geq 50 \mu \mathrm{g}$ of estradiol) [19]. The majority of studies of later-generation OCs containing lower doses of estrogens did not find an increased risk of stroke [20]. However, at least 1 study reported an increased risk of stroke in women using first-, second-, or third-generation OCs [21]. Meta-analyses have also been conflicting [22], and the reasons for the discrepancy are not certain. Fewer data are available on the association between OC use and hemorrhagic stroke. The reported risks appear lower than for ischemic stroke [23], except among older women in whom the risk of hemorrhagic stroke is greatest [24]. Few studies have examined the association between the use of OCs and less common stroke mechanisms. One exception is cerebral venous thrombosis, for which there are data to suggest an association, especially among women with congenital thrombophilias such as factor V Leiden or a prothrombin gene mutation [25].

The absolute increase in stroke risk with low-dose OCs, if one exists, is small. Estimates of the incidence of ischemic stroke in young women range from 0.9 to $\approx 10$ per 100,000 [26]. Using the highest part of this range, even if the risk of stroke is increased by as much as $30 \%$ (ie, 3 per 100,000), the excess number of strokes associated with OC medication remains several-fold lower than the mortality rate for pregnancy in the United States (9 per 100,000 live births) [27]. Some groups of women appear to be at higher risk for stroke associated with OC use. Women who are $>35$ years of age, smoke cigarettes, are hypertensive, are diabetic, have migraines, or have had prior thromboembolic events (especially 
if while on OCs) may be at increased stroke risk if they use OCs. Some data suggest that the total stroke risk may be more than additive for combinations of smoking, migraine with aura, and age $>35$ years, but the issue remains unsettled for low-dose OCs [28].

The risk of stroke associated with OC use is low. Certain women, particularly those who have had a prior thrombotic event, may be at higher risk. Estimates are primarily based on epidemiological studies. The incremental risk of stroke associated with use of low-dose OCs in women without additional risk factors, if one exists, appears low [28]. It is suggested that OCs be discouraged in women with additional risk factors (eg, cigarette smoking or prior thromboembolic events) [29]. For those who elect to assume the increased risk, aggressive therapy of stroke risk factors may be useful.

\section{Antipsychotic Drugs}

A variety of behavioural disturbances such as physical aggression, agitation, hallucinations and wandering commonly accompany dementia. The term behavioural and psychological symptoms of dementia-BPSD-describes this spectrum of non-cognitive manifestations of dementia [30]. The presence of BPSD can decrease quality of life for patients and caregivers, and increases the likelihood of the patient being placed in an institution [31]. Treatment of BPSD is challenging. A variety of non-pharmacological and pharmacological approaches have been assessed [32]. Atypical antipsychotics are often prescribed to manage BPSD. Although such prescriptions represent off-label prescribing, this practise is widely endorsed because atypical antipsychotics are among the best studied treatments for BPSD and there is a perception that they have fewer adverse effects than typical antipsychotics [33].

In April 2005, the U.S. Food and Drug Administration (FDA) issued a public health advisory that the use of atypical antipsychotics to treat elderly patients with dementia was associated with an increased risk for death compared with placebo [34]. In June 2005, Health Canada issued a similar warning and additional data. These warnings stem from reviews of short-term randomized, controlled trials (RCTs) that involve the atypical agents risperidone, olanzapine, quetiapine, and aripiprazole. The mortality rate was approximately 1.6 to 1.7 times higher than with placebo and was greater with antipsychotics than with placebo in 15 of the 17 trials reviewed by the U.S. FDA [35]. The warnings extend to all currently available atypical antipsychotics. Other publications have provided support for these warnings and have raised further safety concerns about older conventional antipsychotics [36-38]. In a recent population-based, retrospective cohort study on older adults with dementia who were followed between 1 April 1997 and 31 March 2003,
Gill [39] conclude that the use of atypical antipsychotics is associated with a small but significant increase in mortality among older adults with dementia. In addition, the risk for death associated with antipsychotics is apparent after as little as 1 month of use and may persist for 6 months. Finally, these data provide independent confirmation of reports that conventional antipsychotics confers an even greater risk for death than does use of atypical antipsychotics $[37,40]$.

The potential causes of death associated with antipsychotic use merit consideration. There was no available information on the proximate causes of death for all patients [41]. Nonetheless, several plausible mechanisms can be proposed. First, antipsychotics may prolong the QT interval, predisposing patients to arrhythmias and sudden cardiac death [42]. Second, sedation and accelerated cognitive decline brought on by exposure to antipsychotics may increase the risk for aspiration syndromes and choking [43]. Aspiration pneumonia is an important cause of death among people with dementia [44]. Third, several studies have found a link between atypical antipsychotic use and venous thromboembolism [45]; therefore, pulmonary embolism may be an underrecognized cause of sudden death in these patients. Fourth, a risk for cerebrovascular events may be associated with antipsychotic use (Table 2).

If atypical agents do contribute to the risk of stroke, however, a plausible biological rationale must exist. The risk seems to develop quickly (6-12 weeks in clinical trials), and thus it seems unlikely that the risk is mediated through drug effects on risk factors such as glucose or lipid metabolism. The metabolic syndrome is highly prevalent in US schizophrenia patients and represents an enormous source of cardiovascular risk, especially for women [46]. Similarly, drug induced atrial fibrillation would be an unlikely explanation for such a rapid increase in the risk of cerebrovascular adverse events. Potential mechanisms for cerebrovascular events related to atypical antipsychotics might include orthostatic hypotension in patients with pre-existing CVD, which might lead to "watershed" strokes [47] and antipsychotic induced hyperprolactinaemia, which might promote platelet aggregation [48]. Others, however, have reported that risperidone may inhibit platelet aggregation (through serotonin receptor antagonism), rather than promote it [49]. Some observational data have shown that antipsychotics might be associated with an increased risk of venous thromboembolic disease [50]; arterial thrombosis such as stroke, however, shares few risk factors with venous thrombosis. A clear biological rationale has not yet been identified for an increased risk of stroke associated with use of atypical antipsychotics. The collection of data on "cerebrovascular adverse events" may have been influenced by patients' receipt of active drug therapy or placebo in the clinical trials. For ex-

Table 2. Potential Causes of Death Associated with Antipsychotic Use

\begin{tabular}{|c|c|}
\hline Antipsychotic use: directly action & Antipsychotic use: indirectly action \\
\hline \hline prolong QT interval & sedation and cognitive decline \\
\hline arhytmias & sedation and aspiration syndrome \\
(pneumonia)
\end{tabular}


ample, it is possible that patients given antipsychotic treatment with relief of psychoses might report more symptoms than patients given placebo [47].

In a recent population based retrospective cohort study Gill [51] identified 32,710 older adults with dementia (17,845 dispensed atypical antipsychotics and 14,865 dispensed typical antipsychotics). In this population based cohort, older adults with BPSD who received atypical antipsychotic drugs seem to have a similar risk of admission to hospital for ischemic stroke as those receiving typical antipsychotic drugs. They conclude that patients dispensed two more consecutive prescriptions (chronic users) of atypical antipsychotics were not at increased risk of stroke compared with chronic users of typical antipsychotics. Finally, the risk of stroke in the subgroup of patients was not significantly different between those receiving atypical antipsychotics and those receiving typical antipsychotics (adjusted hazard ratio $0.98,95 \%$ confidence interval 0.65 to 1.47 ).

Several investigators found no association between use of atypical antipsychotics and cerebrovascular events [52, 53]. In addition, recent data from a trial evaluating quetiapine for BPSD showed no increased risk of cerebrovascular adverse events compared with placebo.

Studies have shown that some patients receiving antipsychotics can be successfully weaned from these medications when monitored closely [54]. Antipsychotic therapies should not be initiated if effective non-drug treatments are available or if symptoms are unlikely to respond to antipsychotic treatment (for example, repetitive vocalizations or wandering).

These findings highlight the need to carefully balance potential risks and benefits when considering antipsychotic treatment for older adults with dementia and emphasize the need to limit use of these drugs to situations in which nonpharmacologic measures have provided an inadequate response.

\section{Thiazolidinediones}

Thiazolidinediones significantly reduce fasting plasma glucose and HbA (1c). These effects are due to improved insulin sensitivity by activating the nuclear PPAR-gamma (peroxisomal proliferator-activated receptors-gamma) and affection of different intracellular functions. Many studies and meta-analyses about thiazolidinediones analysed a significant cardiovascular risk associated with rosiglitazone therapy. Therefore clinicians have been left uncertain as to whether rosiglitazone should still be considered for the treatment of type-2-diabetes. An important side-effect of thiazolidinediones is fluid retention and edema. Therefore, heart failure NYHA I-IV is a contraindication for treatment with thiazolidinediones. Pioglitazone shows favourable changes in lipid parameters like decrease of serumtriglycerides and increase of HDL-cholesterol, while rosiglitazone temporarily increases LDL-cholesterol. In patients with type-2-diabetes mellitus and a high cardiovascular risk the PROactive study did not show a significant effect on the primary endpoint but significantly reduced the predefined secondary combined endpoint of total mortality, nonfatal myocardial infarction and stroke. Conversely, recently published meta-analyses suggested an increased cardiovascular risk and IDH rate associated with rosiglitazone therapy [55].
Treatment with rosiglitazone should be reconsidered because of a potential cardiovascular risk. In high risk patients without heart failure pioglitazone may be favoured for treatment of type 2 diabetes mellitus.

\section{Immunomodulatory Drugs}

Molecular and cellular immune activities have a fundamental role in all stages of the atherosclerotic process and in the pathogenesis of VD. Recent studies have demonstrated how chronic infections can support a local and systemic chronic inflammation, leading to the atherosclerotic process $[56,57]$. The pathogenic link between infection and VD is not completely defined. Some therapeutic strategies, able to influence the persistence of the infections or to modify the inflammatory process, could be useful in primary and secondary vascular disease prevention and in modifying the acute event outcome.

Premature coronary heart disease has emerged as a major cause of morbidity and mortality in systemic autoimmune diseases. Recent epidemiologic and pathogenesis studies have suggested a great deal in common between the pathogenesis of prototypic autoimmune disease such as rheumatoid arthritis and systemic lupus erythematosus and that of atherosclerosis. Some of the most remarkable data in support of a link between autoimmunity and atherosclerosis comes from epidemiological studies of patients with autoimmune disorders (rheumatoid arthritis and systemic lupus erythematosus). Many epidemiologic observations have linked systemic inflammation with the cardiovascular events in autoimmune disease such as rheumatoid arthritis and systemic lupus erythematosus [58]. Systemic levels of soluble inflammatory mediators such as CRP have been associated with cardiovascular risk in the general population. CRP, or more specifically high sensitivity-hsCRP, is a marker of systemic inflammation that has been identified as a valid biomarker of cardiovascular risk. Furthermore, the immunomodulatory and anti-inflammatory actions of statins may affect their utility in the context of chronic inflammatory autoimmune disease. Thus, effective control or dampening of inflammation, with such agents should be included in the therapeutic armamentarium of autoimmune diseases with the aim of protecting against cardiovascular disease [59].

\section{Antiepileptic Drugs}

There is no evidence in literature about the risk of stroke and use of antiepileptic drugs (AED), but in clinical practice an association was found between use of antiepileptic drugs and cerebrovascular events. On the other hand stroke is the leading cause of symptomatic epilepsy in adults, accounting for up to one-third of newly diagnosed seizures among the elderly. About $3 \%$ to $5 \%$ of stroke patients will suffer a remote seizure, $54 \%$ to $66 \%$ of whom will develop epilepsy. Thus far, the optimal timing and type of antiepileptic treatment for patients with post-stroke seizure and epilepsy have not been specifically assessed. Although several studies suggest that seizures alter the functional recovery after a stroke, it remains difficult to determine whether or not the occurrence of a second seizure in an untreated stroke patient might hamper the overall outcome. The decision to initiate AED treatment after a first or a second post-stroke seizure should therefore be individualized, primarily based on the functional impact of the first seizure episode and the patient's prefer- 
ence. Several converging findings suggest that the majority of first-generation AEDs, particularly phenytoin, are not the most appropriate choice in stroke patients because of their potential harmful impact on functional recovery and bone health, their suboptimal pharmacokinetic profile and interaction with anticoagulants or salicylates in modifying therapeutic efficacy in secondary prevention, their greater likelihood of being poorly tolerated, and the lack of level A evidence regarding their specific use in elderly patients. Among the new-generation AEDs that do not interact with anticoagulants, antiplatelet agents, or bone health, lamotrigine and gabapentine are the only two drugs that proved to be more effective than immediate-release carbamazepine in elderly patients, providing level A evidence for their use in this indication. In addition, gabapentin remains the only drug that has been specifically evaluated in stroke patients, demonstrating a high rate of long-term seizure freedom. At present, lowdose lamotrigine or gabapentin appear to represent the optimal first-line therapy for post-stroke seizure and epilepsy in elderly patients or in younger patients requiring anticoagulants. However, low-dose extended-release carbamazepine might be a reasonable and less expensive option in patients with appropriate bone health who do not require anticoagulation [60].

\section{COX-Inhibitors}

The development of drugs that selectively inhibit cyclooxygenase-2 (COX-2) demonstrates translational research from bench to bedside based on underlying knowledge of micro-cellular structure and function. However, theoretical concerns about potentially prothrombotic effects of selective COX-2 inhibitors coupled with observations of increased cardiovascular risk have produced significant consternation and lead to the withdrawal of two of these agents from the market. A number of questions remain unanswered. It appears clear that both selective and non-selective COX inhibitors are associated with increases in blood pressure. In addition, blood pressure is often increased after starting nonsteroidal therapy, and we know that even small increases in blood pressure in subjects with pre-existing vascular disease are associated with substantial increases in the risk of cardiovascular morbidity. Given this line of reasoning, one might hypothesize that the observed increases in the risk of cardiovascular events associated with COX-inhibitors are largely due to increases in blood pressure in populations of subjects who are already at high risk. But can we generalize that the adverse cardiovascular effects observed for rofecoxib and valdecoxib are sufficient to indict the entire class of COX-2 inhibitors, or is this not a class effect, but dependent upon the degree of COX-2 selectivity? In either case, it seems prudent to recommend that subjects who are at higher risk for a cardiovascular event and receiving a COXinhibitor should also be treated with low dose ASA with close follow up of blood pressure and efficacious use of antihypertensive medications. Finally, modest dietary salt restriction may help lessen the effects of COX-inhibitors on blood pressure [61].

\section{HMG-CoA Reductase Inhibitors}

Endothelial dysfunction has been shown to be a prognostic factor for IDH and improvement of endothelial dysfunction prevents cardiovascular event presentation. Endothelial dysfunction is associated to a reduced nitric oxide (NO) bioactivity, as a result of the impairment of NO synthesis/release by the endothelial NO synthase (eNOS) or by inactivation of NO. Endothelial dysfunction measurements are valuable surrogate markers to assess the effectiveness of interventions addressed to prevent or treat ICHD. Dyslipemia and other cardiovascular risk factors promote endothelial dysfunction and life style changes and pharmacological treatment, particularly HMG-CoA reductase inhibitors (statins), have shown early improvement of endothelialdependent vasomotion. Statins efficiently reduce plasma LDL cholesterol, an effect that may account for their beneficial effect on endothelial function, but they also reduce cellular levels of isoprenoid compounds relevant for the bioavailability of NO. Statins restore NO production by several mechanisms, including up-regulation of eNOS mRNA and protein levels and preservation of NO inactivation by reactive oxygen species. These effects are mediated, at least in a part, through mechanisms independent of their lipid lowering effect (pleiotropic effects). The relevance of endotheliumdependent effects on the early and delayed clinical benefit of statins, as well as the multiple ways by which statins may restore endothelial function acting not only on the endothelium but also on endothelial progenitor cells, which probably could contribute to both ischemia-induced neovascularization and endothelial regeneration after injury [62].

Patients with peripheral vascular disease are less likely to receive optimal medical management than patients with coronary artery disease. However, early medical treatment is critical because it is profoundly beneficial and the benefits are maximized. Even in patients with advanced disease requiring invasive intervention, medical management has been proven to improve outcome, prolong the success of the intervention, improve functional capacity, and prolong life. The vascular surgeon should be knowledgeable enough to initiate basic medical therapy and to define for their patients the goals that need to be met to optimize their medical management. The vascular surgeon should be instrumental in assuring that the peripheral vascular patient receives medical therapy of the same standard as the patient with coronary disease. The major modifiable risk factors in the vascular patient are: smoking, high blood pressure, hyperlipidemia, physical inactivity, obesity, and diabetes. In addition, the use of beta blockers for patients with coronary disease and antiplatelet therapy as well as angiotensin-converting enzyme (ACE) inhibitors are recommended for all patients with peripheral vascular disease. Statins have favourable effects on multiple interrelated aspects of vascular biology important in atherosclerosis. In particular they have beneficial effects on inflammation, plaque stabilization, endothelial dysfunction, and thrombosis. Statins have also been shown to be beneficial in acute vascular events. ACE inhibitors have been shown to reduce cardiovascular morbidity and mortality in patients with peripheral arterial disease regardless of the presence or absence of hypertension. A number of the pleiotropic effects of statins are shared by ACE inhibitors. The patients with known vascular disease should be treated aggressively with a combination of a HMG CoA reductase inhibitor, an angiotensin-converting enzyme inhibitor, an antiplatelet agent and a beta blocker if there is a history of coronary disease. They should also receive tight control of their blood pressure and blood sugar. Smokers should be 
encouraged to stop smoking and should be provided with pharmaceutical and emotional support by their physicians. All of these patients should have their body mass index as close to normal as possible and be on a therapeutic lifestyle diet. Regular aerobic exercise is also indicated. Patients with symptomatic claudication should be considered for cilostazol. Patients with multiple risk factors for vascular disease, but who do not have documented disease should also be on statin therapy. As more studies define the linear relationship between lower LDL-C levels and lowered risk of vascular events, indicating that the lower the LDL-C level, the lower the risk, experts are advocating more aggressive lipidlowering therapy. In patients with peripheral arterial disease, some experts now advocate lowering the goal of LDL therapy to $70 \mathrm{mg} / \mathrm{dL}$ [63].

Spondyloarthritides are associated with increased cardiovascular risks, which can only partly be explained by traditional risk factors. It is likely that the chronic inflammatory state is involved. Cardiac pathologies in spondyloarthritides are conduction disturbances and valvular heart diseases. Recent studies have highlighted that the chronic, systemic inflammatory condition of patients with spondyloarthritides may be involved in the development of cardiac and vascular pathologies [64]. Recent data have also focused on vascular pathologies and showed impaired endothelial function, suggesting that atherosclerotic alterations could also be involved in increased cardiovascular mortality.

\section{DRUG ABUSE}

Drugs of abuse, including cocaine, amphetamines, and heroin, have been associated with an increased risk of VD (65); drugs of abuse increased the risk of stroke 6.5-fold (95\% CI 3.1 to 13.6 ) across all age groups and with an RR of $11.2(95 \%$ CI 3.2 to 42.5 ) in persons < 35 years of age [66].

Drug addiction is often a chronic relapsing disorder associated with a number of societal and health-related problems [67]. These drugs can cause abrupt changes in blood pressure, induce vasculitic-type changes, lead to embolization caused by infective endocarditis and induce hemostatic and hematologic abnormalities that can result in increased blood viscosity and platelet aggregation [1]. Information about stroke-related drug abuse is mainly limited to epidemiological studies focused on urban populations. An increase in the risk of both ischemic and hemorrhagic stroke has been reported [68]. In one study, drug abuse increased the risk of stroke 6.5 -fold (95\% CI 3.1 to 13.6) across all age groups and with an RR of 11.2 (95\% CI 3.2 to 42.5 ) in persons $<35$ years of age. Long-term treatment strategies based on medication, psychological support, and outreach programs play an important part in treatment of drug dependency [69].

Drug abuse is associated with a variety of neurological complications. The use of certain recreational drugs shows a marked temporal association with the onset of both haemorrhagic and ischemic strokes, the majority of which develop within minutes to one hour after the administration of the index drug. Delayed onset of stroke has also been observed. Acute, severe elevation of blood pressure, cardiac dysrhythmias, cerebral vasospasm, vasculitis, embolization due to infective endocarditis or dilated cardiomyopathy, embolization due to foreign material injected with the diluents under non-sterile conditions and 'street drug' contaminants with cardiovascular effects have been suggested as possible underlying mechanisms. Rupture of aneurysms and arteriovenous malformations have been detected in up to half of the patients with haemorrhagic stroke due to cocaine abuse. The less common findings reported have included a mycotic cerebrovascular aneurysm in a patient with infective endocarditis and haemorrhagic stroke. In addition to stroke, cocaine seems to provoke vascular headache. Seizures precipitated by recreational drug abuse are usually caused by acute intoxication in contrast to the withdrawal seizures encountered in subjects with alcohol abuse. Movement disorders and cerebral atrophy correlating with the duration of abuse have been described. Snorting of organic solvents may cause encephalopathy. Cases of spongiform leukoencephalopathy in heroin addicts have also been reported. Peripheral neuropathy is occasionally precipitated by drug poisoning after intravenous administration. Impurities of the drug, risky administration techniques, and the use of mixtures of various drugs, frequently with simultaneous alcohol drinking, should be taken into account when assessing the background of the adverse event as well as the overall lifestyle of the addicted subjects [1].

Cannabinoid drugs exert their effects primarily through activation of cannabinoid $\mathrm{CB} 1$ and $\mathrm{CB} 2$ receptors. Both $\mathrm{CB} 1$ and $\mathrm{CB} 2$ receptors have been implicated in a number of cardiovascular processes, including vasodilation, cardiac protection, modulation of the baroreceptor reflex in the control of systolic blood pressure, and inhibition of endothelial inflammation and the progress of atherosclerosis in a murine model. These effects are mainly mediated through central and peripheral nervous system $\mathrm{CB} 1$ receptors, vascular $\mathrm{CB} 1$ receptors and immune cell $\mathrm{CB} 2$ receptors. Relevant cellular effects include: the inhibition of neurotransmitter release in the nucleus tractus solitarius and in peripheral adrenergic neurons; regulation of NOS activity in vascular beds; inhibition of vascular smooth muscle cell excitability; regulation of endothelial cell migration and proliferation; and effects on immune cell proliferation, activation, and inflammatory functions [70].

Several drugs of abuse have been associated with stroke. However, there is a paucity of data on the independent risk of stroke associated with drugs of abuse and no controlled trials demonstrating a reduction in risk with abstinence. Successful identification and management of drug abuse can be challenging. When a patient is identified as having a drug addiction problem, referral for appropriate counselling may be considered.

\section{CONCLUSION}

Experimental data, clinical investigations and epidemiological studies identified the association of some pharmacological treatment and the several drugs of abuse with VD. These findings highlight the need to carefully balance potential risk and benefits when considering pharmacological treatment in young and older people. The results of these findings represent a scientific and clinical necessity so as to identify a new group of "modifiable" risk factors to improve VD prevention. Further safety pharmacological studies should focus on identifying the pathophysiology mechanisms of vascular risk and determining whether and how different 
regimens of pharmacological treatment and the abuse of several drugs influence VD.

\section{REFERENCES}

[1] Neiman J, Haapaniemi H.M. and Hillbom M. Neurological complications of drug abuse: Pathophysiological mechanisms. Eur J Neurol 2000 7; 6: 595-606.

[2] Shenouda SM, Vita JA. Effects of flavonoid-containing beverages and EGCG on endothelial function. Am Coll Nutr 2007; 26(4): 366S-372S.

[3] Lowe GD. The relationship between infection, inflammation, and cardiovascular disease: an overview. Ann Periodontol 2001; 6(1): $1-8$.

[4] Mosca L, Collins P, Herrington DM, et al. Hormone replacement therapy and cardiovascular disease. A statement for healthcare professionals from the AHA. Circulation 2001; 104: 499-503.

[5] Kalantaridou SN, Naka KK, Papanikolaou E, et al. Impaired endothelial function in young women with premature ovarian failure: normalization with hormone therapy. J Clin Endocrinol Metab 2004; 89: 3907-13.

[6] Subbiah MR. Estrogen replacement therapy and cardioprotection: mechanisms and controversies. Braz J Med Biol Res 2002; 35: 271-76.

[7] Khalil RA. Sex hormones as potential modulators of vascular function in hypertension. Hypertension 2005; 46: 249-54.

[8] Hermenegildo C, Oviedo PJ, Cano A. Cyclooxygenases regulation by estradiol on endothelium. Curr Pharm Des 2006; 12: 205-15.

[9] Nilsson S, Gustafsson JA. Estrogen receptor transcription and transactivation. Basic aspects of estrogen action. Breast Cancer Res 2000; 2: 360-6.

[10] Barchiese F, Jackson EK, Gillespie DJ, Zacharia LC, Fingerle J, Dubbey RK. Methoxyestradiols mediate estradiol-induced antimitogenesis in human aortic SMCs. Hypertension 2002; 39: 874-9.

[11] Pare Gary, Krust A, Karas R, et al. Estrogen receptor-a mediates the protective effects of estrogen against vascular injury. Circ Res 2002; 90: 1087-92.

[12] Scheuer J, Malhotra A, Schaible TF, Capasso J. Effects of gonadectomy and hormonal replacement on the rat hearts. Circ Res 1987; 61: 12-9.

[13] Sutton-Tyrrell K, Wildman RP, Matthews KA, et al. Sex hormonebinding globulin and the free androgen index are related to cardiovascular risk factors in multiethnic premenopausal and perimenopausal women enrolled in the Study of Women Across the Nation (SWAN). Circulation 2005; 111: 1242-9.

[14] Ding EL, Song Y, Maliki VS, Liu S. Sex differences of endogenous sex hormones and risk of type 2 diabetes: a systematic review and metaanalysis. JAMA 2006; 295: 1288-99.

[15] Maturana MA and Spritzer PM. Association between hyperinsulinemia and endogenous androgen levels in peri- and postmenopausal women. Metabolism 2002; 51: 238-43.

[16] Rosano GM, Vitale C, Marazzi G, Volterrani M. Menopause and cardiovascular disease: the evidence. Climacteric 2007; 10 Suppl 1: 19-24.

[17] Farley TM, Meirik O, Chang CL, Poulter NR. Combined oral contraceptives, smoking, and cardiovascular risk. J Epidemiol Commun Health 1998; 52: 775-785.

[18] Cushman M, Kuller LH, Prentice R, et al.Women's Health Initiative Investigators. Estrogen plus progestin and risk of venous thrombosis. JAMA 2004 6; 292(13): 1573-80.

[19] Bushnell CD, Goldstein LB. Ischemic stroke: recognizing risks unique to women. Womens Health Primary Care 1999; 2: 788-804.

[20] Chan WS, Ray J, Wai EK, Ginsburg S, Hannah ME, Corey PN, Ginsberg JS. Risk of stroke in women exposed to low-dose oral contraceptives: a critical evaluation of the evidence. Arch Intern Med 2004; 164: 741-747.

[21] Heinemann LA, Lewis MA, Thorogood M, Spitzer WO, Guggenmoos-Holzmann I, Bruppacher R. Case-control study of oral contraceptives and risk of thromboembolic stroke: results from international study on oral contraceptives and health of young women. BMJ 1997; 315: 1502-1504.

[22] Gillum LA, Mamidipudi SK, Johnston SC. Ischemic stroke risk with oral contraceptives: a meta-analysis. JAMA 2000; 284: 72-78.

[23] Johnston SC, Colford JM Jr, Gress DR. Oral contraceptives and the risk of subarachnoid hemorrhage: a meta-analysis. Neurology1998; 51: 411-418
[24] World Health Organization Scientific Group on Cardiovascular Disease and Steroid Hormone Contraception. Cardiovascular disease and steroid hormone contraception: report of the World Health Organization Scientific Group. Geneva, Switzerland: World Health Organization; 1998.

[25] Martinelli I, Sacchi E, Landi G, Taioli E, Duca F, Mannucci PM. High risk of cerebral-vein thrombosis in carriers of a prothrombingene mutation and in users of oral contraceptives. N Engl J Med 1998; 338: 1793-1797.

[26] Nightingale AL, Farmer RD. Ischemic stroke in young women: a nested case-control study using the UK General Practice Research Database. Stroke 2004; 35: 1574-1578.

[27] Hoyert DL, Arias E, Smith BL, Murphy SL, Kochanek KD. Deaths: final data for 1999. Natl Vital Stat Rep 2001; 49: 1-113.

[28] Siritho S, Thrift AG, McNeil JJ, You RX, Davis SM, Donnan GA; Melbourne Risk Factor Study (MERFS) Group. Risk of ischemic stroke among users of the oral contraceptive pill: the Melbourne Risk Factor Study (MERFS) Group. Stroke 2003; 34: 1575-1580.

[29] Bousser MG, Kittner SJ.Oral contraceptives and stroke. Cephalalgia 2000; 20(3): 183-9.

[30] Cohen-Mansfield J, Billig N. Agitated behaviours in the elderly. I: a conceptual review. J Am Geriatr Soc 1998; 36: 7-12.

[31] Cohen CA, Gold DP, Shulman KI,Wortley JT, McDonald G,Wargon M. Factors determining the decision to institutionalize dementing individuals: a prospective study. Gerontologist 1993; 33: 714-20

[32] Teri L, Logsdon RG, McCurry SM. Nonpharmacologic treatment of behavioral disturbance in dementia. Med Clin North Am 2002; 86: 641-56.

[33] Tariot PN, Ryan JM, Porsteinsson AP, Loy R, Schneider LS. Pharmacologic therapy for behavioral symptoms of Alzheimer's disease. Clin Geriatr Med 2001; 17: 359-76.

[34] U.S. Food and Drug Administration. FDA issues public health advisory for antipsychotic drugs used for treatment of behavioral disorders in elderly patients. FDA Talk Paper T05-13. Rockville, MD: U.S. Food and Drug Administration; 11 April 2005.

[35] Khan A, Schwartz K, Stern C, Redding N, Kolts RL, Brown WA, Robinson DS. Mortality risk in patients with schizophrenia participating in premarketing atypical antipsychotic clinical trials. J Clin Psychiatry 2007; 68(12): 1828-33.

[36] Schneider LS, Dagerman KS, Insel P. Risk of death with atypical antipsychotic drug treatment for dementia: meta-analysis of randomized placebocontrolled trials. JAMA 2005; 294: 1934-43.

[37] Wang PS, Schneeweiss S, Avorn J, et al. Risk of death in elderly users of conventional vs. atypical antipsychotic medications. N Engl J Med 2005; 353: 2335-41.

[38] Kryzhanovskaya LA, Jeste DV, Young CA, et al. A review of treatment-emergent adverse events during olanzapine clinical trials in elderly patients with dementia. J Clin Psychiatry 2006; 67: 93345.

[39] Gill SS, Bronskill SE, Normand SL, et al. Antipsychotic drug use and mortality in older adults with dementia. Ann Intern Med 2007; 146: 775-786.

[40] Rabins PV, Lyketsos CG. Antipsychotic drugs in dementia: what should be made of the risks? [Editorial]. JAMA 2005; 294: 1963-5.

[41] Ray WA. Observational studies of drugs and mortality. N Engl J Med 2005; 353: 2319-21.

[42] Liperoti R, Gambassi G, Lapane KL, et al. Cerebrovascular events among elderly nursing home patients treated with conventional or atypical antipsychotics. J Clin Psychiatry 2005; 66: 1090-6.

[43] Warner J. Risk of choking in mental illness. Lancet 2004; 363: 674.

[44] Keene J, Hope T, Fairburn CG, Jacoby R. Death and dementia. Int J Geriatr Psychiatry 2001; 16(10): 969-74.

[45] Liperoti R, Gambassi G, Lapane KL, et al. Conventional and atypical antipsychotics and the risk of hospitalization for ventricular arrhythmias or cardiac arrest. Arch Intern Med 2005; 165: 696-701.

[46] McEvoy JP, Meyer JM, Goff DC, et al. Prevalence of the metabolic syndrome in patients with schizophrenia: baseline results from the Clinical Antipsychotic Trials of Intervention Effectiveness (CATIE) schizophrenia trial and comparison with national estimates from NHANES III. Schizophr Res 2005; 80(1): 19-32.

[47] Smith DA, Beier MT. Association between risperidone treatment and cerebrovascular adverse events: examining the evidence and postulating hypotheses for an underlying mechanism. J Am Med Dir Assoc 2004; 5: 129-32. 
[48] Wallaschofski H, Donne M, Eigenthaler M, et al. PRL as a novel potent cofactor for platelet aggregation. J Clin Endocrinol Metab 2001; 86: 5912-9.

[49] Harrison-Woolrych M, Clark DWJ. Nose bleeds associated with use of risperidone. BMJ 2004; 328: 1416.

[50] Zornberg GL, Jick H. Antipsychotic drug use and risk of first-time idiopathic venous thromboembolism: a case-control study. Lancet 2000; 356: 1219-23

[51] Gill SS, Rochon PA, Herrmann N, et al. Atypical antipsychotic drugs and risk of ischemic stroke: population based retrospective cohort study. BMJ 2005; 330: 445.

[52] Herrmann N, Mamdani M, Lanctôt KL. Atypical antipsychotics and risk of cerebrovascular accidents. Am J Psychiatry 2004; 161: 1113-5.

[53] Liperoti R. Cerebrovascular events among elderly patients treated with conventional or atypical antipsychotics. Annual meeting of the American Geriatrics Society 2004.

[54] van Reekum R, Clarke D, Conn D, et al. A randomized, placebocontrolled trial of the discontinuation of long-term antipsychotics in dementia. Int Psychogeriatr 2002; 14: 197-210.

[55] Rottlaender D, Michels G, Erdmann E, Hoppe UC. Therapy with glitazones a risk for cardiovascular disease? Dtsch Med Wochenschr 2007; 132(49): 2629-32.

[56] Matsuura E, Kobayashi K, Lopez LR. Preventing autoimmune and infection triggered atherosclerosis for an enduring healthful lifestyle. Autoimmun Rev 2008; 7(3): 214-22.

[57] Mallika V, Goswami B, Rajappa M. Atherosclerosis pathophysiology and the role of novel risk factors: a clinicobiochemical perspective. Angiology 2007; 58(5): 513-22.

[58] Zinger H, Sherer Y, Shoenfeld Y. Atherosclerosis in autoimmune rheumatic diseases-mechanisms and clinical findings. Clin Rev Allergy Immunol 2009; 37(1): 20-8.
[59] Abou-Raya A, Abou-Raya S. Inflammation: a pivotal link between autoimmune diseases and atherosclerosis. Autoimmun Rev 2006; 5(5): 331-7; 2006

[60] Ryvlin P, Montavont A, Nighoghossian N. Optimizing therapy of seizures in stroke patients. Neurology 2006; 67(12 Suppl 4): S3-9.

[61] Salzberg DJ, Weir MR. COX-2 inhibitors and cardiovascular risk Subcell Biochem 2007; 42: 159-74.

[62] Martínez-González J, Badimon L. Influence of statin use on endothelial function: from bench to clinics. Curr Pharm Des 2007; 13(17): 1771-86

[63] Rice TW, Lumsden AB. Optimal medical management of peripheral arterial disease. Vasc Endovasc Surg 2006; 40(4): 312-27.

[64] Heeneman S, Daemen MJ. Cardiovascular risks in spondyloarthritides. Curr Opin Rheumatol 2007; 19(4): 358-62.

[65] Brust JCM. Neurological Aspects of Substance Abuse. 2nd ed Philadelphia, Butterworth-Heinemann; 2004.

[66] Kaku DA, Lowenstein DH. Emergence of recreational drug abuse as a major risk factor for stroke in young adults. Ann Intern Med 1990; 113: 821-827.

[67] Cami J, Farre M. Drug addiction. N Engl J Med 2003; 349: 975986.

[68] Kittner SJ, Stern BJ, Wozniak M, et al. Cerebral infarction in young adults: the Baltimore-Washington Cooperative Young Stroke Study. Neurology 1998; 50: 890-894

[69] Johnson K, Noe T, Collins D, et al. Mobilizing church communities to prevent alcohol and other drug abuse: a model strategy and its evaluation. J Commun Pract 2000; 7: 1-27.

[70] Ashton JC, Smith PF. Cannabinoids and cardiovascular disease: the outlook for clinical treatments. Curr Vasc Pharmacol 2007; 5(3): 175-85.

(C) Bacigaluppi et al.; Licensee Bentham Open.

This is an open access article licensed under the terms of the Creative Commons Attribution Non-Commercial License (http://creativecommons.org/licenses/by-nc/3.0/) which permits unrestricted, non-commercial use, distribution and reproduction in any medium, provided the work is properly cited. 